\title{
Alignment Signal Aided CP-Free SEFDM
}

\author{
Bo Sun*, Waseem Ozan ${ }^{\dagger}$, Toni Levanen*, Bo Tan*, Markku Renfors*, Izzat Darwazeh ${ }^{\dagger}$, and Mikko Valkama* \\ * Faculty of Information Technology and Communication Sciences, Tampere University, Finland \\ $\dagger$ Department of Electronics and Electrical Engineering, University College London, UK \\ \{bo.sun, bo.tan, toni.levanen, mikko.valkama\}@tuni.fi; \{w.ozan, i.darwazeh\}@ucl.ac.uk
}

\begin{abstract}
This paper proposes a cyclic prefix (CP) free spectral efficiency frequency division multiplexing (SEFDM) wireless signal transmission and reception method based on alignment signal (AS). The method needs the cooperation of both the transmission and reception sides. At the transmitter, time-domain AS is designed to prevent inter-symbol interference (ISI) caused by multipath propagation in the received signal. At the receiver, the channel circularity convolution providing processing is used to enable high accurate frequency domain one-tap equaliser. The extensive computer simulation results under the $5 \mathrm{G}$ new radio (5G-NR) channel model, TDL$D$, show the that the AS aided SEFDM has similar bit error rate performance as CP-SEFDM without energy and latency cost on CP. The AS-SEFDM capability to mitigate the ISI and to enable the one-tap equaliser in SEFDM systems makes it a promising technique for future wireless communication systems.
\end{abstract}

Index Terms - cyclic prefix free, spectral efficiency frequency division multiplexing, alignment signal, inter-symbol interference, beyond 5G communications

\section{INTRODUCTION}

Higher spectral efficiency and flexible sub-bands operating are always key objectives in the wireless communications research for achieving higher system capacity, adaptable user accessing, as well as combating the multipath fading. Guard interval aided orthogonal frequency division multiplexing (GI-OFDM) [1], including cyclic prefix (CP)-OFDM and zero-padding (ZP)-OFDM are well-known signalling scheme for above objectives. Specially, CP-OFDM has used in fifth generation (5G) [2], long-term evolution (LTE) [3], and 802.11x [4]. The CP-OFDM connects the a duplicated segment from the OFDM symbol end to head for counteracting ISI caused by multipath and providing circularity which makes simple one-tap frequency domain channel equalisation available.

The concept of using CP part in the transmitted signal was challenged in a classic paper published in 2005 [5]. In which, it was demonstrated that the effects of ISI can be cancelled at the receiver, yet no $\mathrm{CP}$ part was needed in the transmitted signal. This debate of whether using a GI or not was revisited in 2018 [6], yet this time considering the use of an alignment signal (AS), which is added on top of the transmitted symbol in the time-domain. Thus, the received symbols are ISI-free, also, the channel convolution matrix is modelled as a circulant matrix.

This widely accepted paradigm of using OFDM signals for multi-carrier systems, however, has been challenged in the recent years where non-orthogonal signals (in particular spectral efficient frequency division multiplexing (SEFDM)) is considered to offer higher spectral efficiency in comparison to OFDM ones. SEFDM saves the signal bandwidth by compressing the frequency spacing between the subcarriers and hence enhancing the spectral efficiency [7], which makes it a topic of current interest [8]-[12].

Similar as the OFDM signal, CP can be used for mitigate the ISI in the SEFDM signal. However, in CP-SEFDM signals, there is a discontinuity in phase between the $\mathrm{CP}$ portion and the SEFDM symbol leads to interference being introduced from the CP to the SEFDM symbol [13]. Thus, after removing the CP portion at the receiver, the SEFDM symbols are padded with zeros at the input of the discrete Fourier transform (DFT), which in turn yields a non-continuous signal in the time-domain, hence introducing additional interference to the subcarriers. As a result, CP-SEFDM often suffers the inaccurate channel equalisation because of the non-zero mean square error (MSE) floor in the channel estimation which has been reported in [13]. In addition to symbol interference introduced by $\mathrm{CP}$, the CP-SEFDM also suffers the coarse channel estimation and equalisation caused by non-orthogonality between the subcarriers, which is also known as inter-carrier interference (ICI). To address this issue in CP-SEFDM, researchers have tried time-domain channel estimation and equalisation [14], OFDM pilot symbols for channel estimation [15]-[17]. The solutions either cause high time-domain processing complexity or do not bring clear improvement in channel equalisation. Recently, ZP-SEFDM is proposed in [8], [13] to provide accurate channel estimation and equalisation performance in the frequency-domain.

Nevertheless, all the previous SEFDM techniques rely on the use of either CP or ZP to mitigate the ISI, which reduces the spectral efficiency of the overall system. Besides the general overheads, GI duration, which is often assumably determined by the maximum channel delay spread, blocks the flexible waveform parameterisation. This fact contradicts with the future wireless communications trend. For example, 5G-NR allows mixed-numerology operation which uses different subcarrier spacing and $\mathrm{CP}$ duration in different sub-bands [18]. The CP duration in 5G-new radio (5GNR) scales directly with the chosen subcarrier spacing. This fact makes CP duration have limited choice also increases the system parameterisation in mixed-numerology scenario. Thus, we propose a CP-free SEFDM based on the use of 
AS in this work. The new AS-SEFDM technique has the advantage of ISI self-cancellation, also, it enables modelling the channel as a circulant matrix. Thus, enables the use of one-tap equaliser in the frequency-domain. In addition, the newly proposed mechanism enjoys the extra savings in energy and improved spectral efficiency coming from the CP-free part, simplify the system parameterisation and reduce the physical layer latency as more SEFDM symbols can be transmitted within the same period which is in favour of $6 \mathrm{G}$ low-latency use cases.

The rest of the paper is organised as the following: Section II introduces the SEFDM signal model, the AS design and circularity providing processing; the system performance evaluation of AS-SEFDM is compared with CP-SEFDM and CP-OFDM schemes in Section III; at last the, the conclusion is given in Section IV.

\section{SignAl REPRESENTATION AND MODELLING OF CP-SEFDM AND AS-SEFDM}

In this section, SEFDM signal modelling is given in Section II-A, and signal representation and modelling of the CP-SEFDM scheme is provided in Section II-B, while ASSEFDM scheme is given in Section II-C.

\section{A. SEFDM Signal Model}

SEFDM is a multi-carrier signal, which is generated by modulating $N$ subcarriers with $N$ complex symbols. The frequency spacing between the subcarrier is lower than that of the OFDM signal by a factor $\alpha$, where $\alpha$ is the bandwidth compression factor, in comparison to OFDM. Thus, SEFDM offers bandwidth saving equal to $((1-\alpha) \times$ total bandwidth) in Hz. An SEFDM symbol, $\mathbf{x} \in \mathbb{C}^{Q \times 1}$, is mathematically given as:

$$
x(k)=1 / \sqrt{Q} \times \sum_{n=0}^{Q-1} s_{n} e^{((j 2 \pi \alpha n k) / Q)}
$$

where $k=[0,1, \ldots, Q-1]$ denotes the time samples index in one SEFDM symbol, $Q$ is the size of the inverse discrete Fourier transform (IDFT)/DFT processes and it is equal to $Q=\rho N$, where $\rho$ is the oversampling rate and $s_{n}$ is the complex symbol modulated on the $n^{\text {th }}$ subcarrier.

\section{B. $C P-S E F D M$}

The CP-SEFDM signal structure is illustrated in Fig. 1. In which, steps 1 and 2 depict the output of the IDFT process, the discarded time samples and the resulting SEFDM symbol in the time-domain. In the same figure, step 3 depicts the conventional CP-SEFDM signal generation. The CP part in CP-SEFDM is generated by copying the last $\mu \in \mathbb{N}$ time samples of an SEFDM symbol, $\overline{\mathbf{x}}_{c p} \in \mathbb{C}^{\mu \times 1}$ and attaching them to the beginning of the transmitted SEFDM symbol. After that, the CP-SEFDM symbol is passed through a wireless multipath channel with channel impulse response (CIR) $\mathbf{h}=\left[h_{0}, h_{1}, \ldots, h_{\mu}\right] \in \mathbb{C}^{(\mu+1) \times 1}$. It should be noted that, unlike CP-OFDM symbols, CP-SEFDM symbols suffer of phase discontinuity between the CP part and the symbol [13].

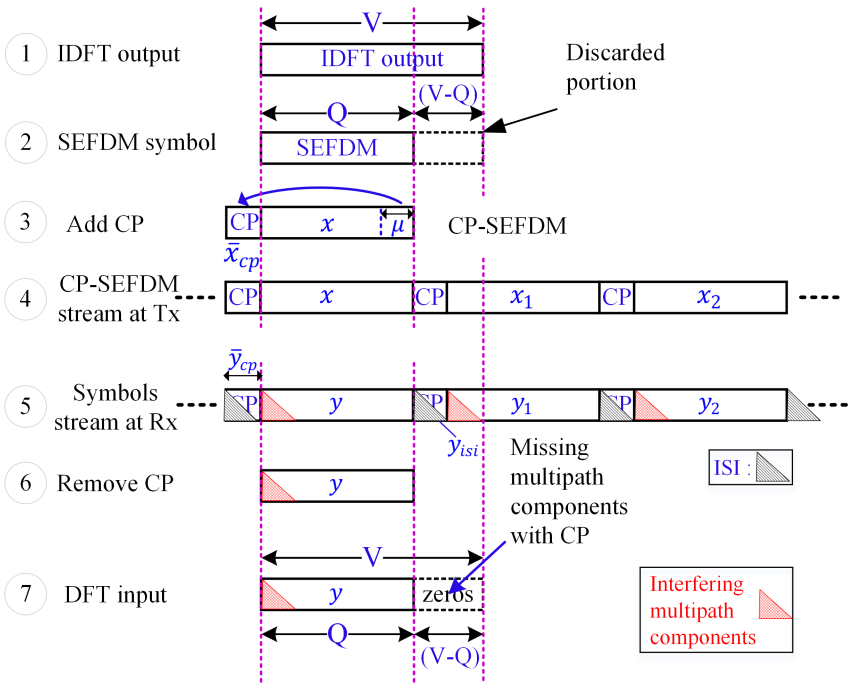

Fig. 1. Signal structure representation for CP-SEFDM.

Each received CP-SEFDM symbol consists of three parts, $\overline{\mathbf{y}}_{c p}, \mathbf{y}$ and $\mathbf{y}_{i s i}$, as depicted in step 5 in Fig. 1. The first part, $\overline{\mathbf{y}}_{c p} \in \mathbb{C}^{\mu \times 1}$, represents the CP part, which is affected by the ISI components coming from the previous symbol and hence it is neglected at the receiver; while the second part, $\mathbf{y} \in \mathbb{C}^{Q \times 1}$, denotes the received SEFDM symbol that is passed to the detection stage; and finally the third part, $\mathbf{y}_{i s i} \in \mathbb{C}^{\mu \times 1}$, is the ISI components, which are added to the succeeding symbol. Having removed the $\mathrm{CP}$, the demodulated CP-SEFDM symbol, $\tilde{\mathbf{r}}_{c p}$, will be given as [13]:

$$
\tilde{\mathbf{r}}_{c p}=\boldsymbol{\lambda} \odot \tilde{\mathbf{s}}-\tilde{\mathbf{r}}_{c p 2}+\tilde{\mathbf{r}}_{c p 3}+\tilde{\mathbf{z}}_{c p}
$$

where $\boldsymbol{\lambda}$ is the subchannel gains vector, $\tilde{\mathbf{s}}=\mathbf{C s}$ is the expected received SEFDM symbol when there is no multipath channel effects or noise channel, $\mathbf{C}=\boldsymbol{\Phi}^{H} \boldsymbol{\Phi}$ is the correlation matrix, which models the interference coefficients of each subcarrier to its neighbouring subcarriers [19], the element $\tilde{\mathbf{z}}_{c p}$ is the additive white Gaussian noise (AWGN) noise vector, and the notation symbol $(\odot)$ is the element-wise multiplication. It should be evident that even in the absence of noise, the demodulated signal would carry additional interference components from the unwanted and missing signals, $\tilde{\mathbf{r}}_{c p 2}$ and $\tilde{\mathbf{r}}_{c p 3}$, respectively.

Channel Equalisation in CP-SEFDM: The channel equalisation analytical expression of CP-SEFDM is found using one-tap equaliser and it is given as [13]:

$$
\begin{aligned}
\hat{\mathbf{r}}_{c p} & =\tilde{\mathbf{r}}_{c p} \cdot / \boldsymbol{\lambda}=\left(\boldsymbol{\lambda} \odot \tilde{\mathbf{s}}-\tilde{\mathbf{r}}_{c p 2}+\tilde{\mathbf{r}}_{c p 3}+\tilde{\mathbf{z}}_{c p}\right) \cdot / \boldsymbol{\lambda} \\
& =\underbrace{\tilde{\mathbf{s}}}_{1}-\underbrace{\tilde{\mathbf{r}}_{c p 2} \cdot / \boldsymbol{\lambda}}_{2}+\underbrace{\tilde{\mathbf{r}}_{c p 3} \cdot / \boldsymbol{\lambda}}_{3}+\underbrace{\tilde{\mathbf{z}}_{c p} \cdot / \boldsymbol{\lambda}}_{4}
\end{aligned}
$$

where part 1 is the desired signal, parts 2 and 3 are the added interference, part 4 corresponds to the noise signal [13]. The full derivations of the analytical expressions for CP-SEFDM can be found in [13]. 


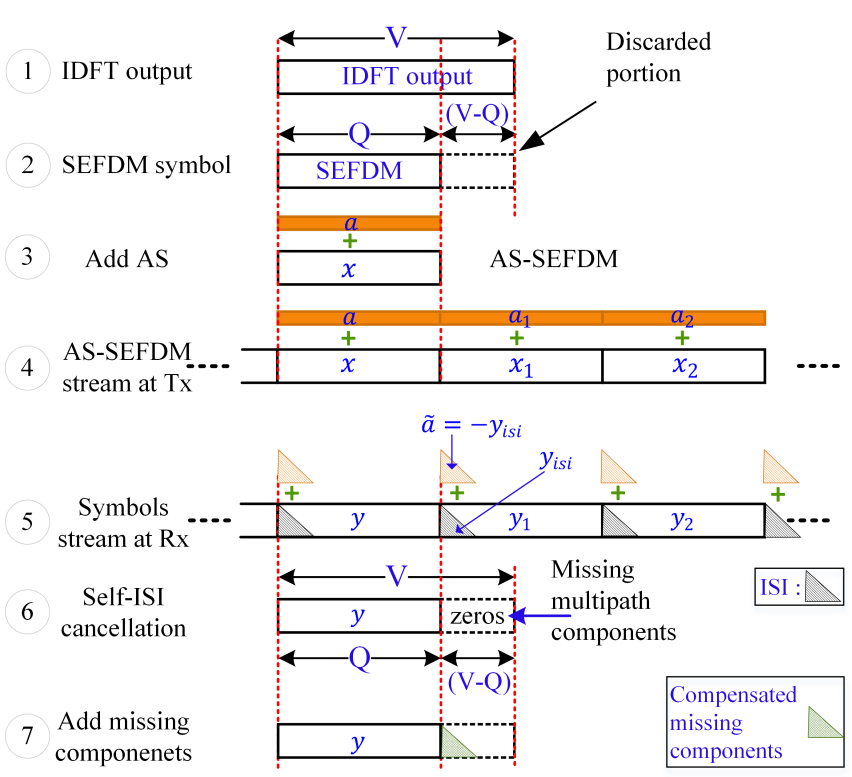

Fig. 2. Signal structure representation for AS-SEFDM.

\section{C. $A S-S E F D M$}

Here, we propose a new scheme of SEFDM signals, which is a CP-less SEFDM with AS. Unlike CP-SEFDM, the new AS-SEFDM signal has no CP part in its transmitted symbols. However, to enable the one-tap equalisation process, the received signal before equalisation must meet the two conditions: $(i)$ being ISI-free and (ii) provide channel circularity. To achieve this, the AS-SEFDM scheme is obtained by generating and combining two signals with SEFDM signals. These two signals are: $(i)$ the ISI-cancelling signal, and $(i i)$ the circularity-providing signal. The first signal is added on top of the transmitted signal, as shown in steps 3 and 4 in Fig. 2, thus the received signal is ISI-free, as shown in steps 5 and 6 in Fig. 2. The second signal is added at the receiver in the time-domain, as shown in steps 7 in Fig. 2, to provide channel circularity.

1) The ISI-cancelling signal: is expressed by $\mathbf{a}_{\mathbf{i}} \in \mathbb{C}^{Q \times 1}$, and is generated at the transmitter side and added on top of the $i^{\text {th }}$ SEFDM symbol in the time-domain, as shown in steps 3 and 4 in Fig. 2. Thus, the received AS-SEFDM signal, after traversing a multipath channel, has no ISI. In order to find the analytical expression of the ISI-cancelling signal, we do the following.

At the transmitter, the $i^{\text {th }}$ AS-SEFDM symbol is represented as:

$$
\mathbf{x}_{\mathbf{i} a s}=\mathbf{x}_{\mathbf{i}}+\mathbf{a}_{\mathbf{i}}
$$

where $\mathbf{x}_{\mathbf{i}}$ is the $i^{\text {th }}$ SEFDM symbol vector and $\mathbf{a}_{\mathbf{i}}$ is the $i^{t h}$ ISI-cancelling signal vector. The AS-SEFDM symbol, $\mathbf{x}_{\mathbf{i} a s} \in \mathbb{C}^{(Q) \times 1}$, after traversing a multipath channel, with channel impulse response $\left(\mathbf{h}=\left[h_{0}, h_{1}, \ldots, h_{\mu}\right] \in\right.$ $\left.\mathbb{C}^{(\mu+1) \times 1}\right)$, is given by:

$$
\mathbf{y}_{\mathbf{i}}=\mathbf{h} * \mathbf{x}_{\mathbf{i}_{a s}}=\mathbf{h} * \mathbf{x}_{\mathbf{i}}+\mathbf{h} * \mathbf{a}_{\mathbf{i}}=\mathbf{H} \mathbf{x}_{\mathbf{i}}+\mathbf{H a}_{\mathbf{i}}
$$

where $\mathbf{H} \in \mathbb{C}^{(Q+\mu) \times Q}$ is the convolution channel matrix and it is given as:

$$
\mathbf{H}=\left[\begin{array}{c}
\mathbf{H}_{u} \\
\mathbf{H}_{l}
\end{array}\right]=\left[\begin{array}{cccccc}
h_{0} & 0 & \ldots & \ldots & \ldots & 0 \\
h_{1} & h_{0} & \ddots & & & \vdots \\
\vdots & h_{1} & \ddots & \ddots & & \vdots \\
h_{\mu} & \vdots & \ddots & h_{0} & \ddots & \vdots \\
0 & h_{\mu} & \ddots & h_{1} & \ddots & 0 \\
\vdots & \ddots & \ddots & \vdots & \ddots & h_{0} \\
\vdots & & \ddots & h_{\mu} & \ddots & h_{1} \\
\vdots & & & \ddots & \ddots & \vdots \\
0 & \ldots & \ldots & \ldots & 0 & h_{\mu}
\end{array}\right]
$$

where $\mathbf{H}_{u} \in \mathbb{C}^{Q \times Q}$ and $\mathbf{H}_{l} \in \mathbb{C}^{\mu \times Q}$ are the upper and lower parts of the channel matrix, respectively. Therefore, (5) can be re-written as:

$$
\mathbf{y}_{\mathbf{i}}=\left[\begin{array}{c}
\mathbf{H}_{u} \\
\mathbf{H}_{l}
\end{array}\right] \mathbf{x}_{\mathbf{i}}+\mathbf{H a}_{\mathbf{i}}=\underbrace{\left[\begin{array}{c}
\mathbf{H}_{u} \\
\mathbf{0}_{\mu \times 1}
\end{array}\right] \mathbf{x}_{\mathbf{i}}}_{1}+\underbrace{\left[\begin{array}{c}
\mathbf{0}_{Q \times 1} \\
\mathbf{H}_{l}
\end{array}\right] \mathbf{x}_{\mathbf{i}}}_{2}+\underbrace{\mathbf{H a}_{\mathbf{i}}}_{3}
$$

where part 1 represents the SEFDM symbol affected by the multipath and part 2 is the ISI signal stemming from the $i^{t h}$ SEFDM symbol, which is added to the next $(i+1)^{t h}$ symbol, while part 3 is the ISI-cancelling signal after passing a multipath channel.

To get a received SEFDM symbol with ISI-free, the values of the signal given in parts 2 and 3 must be equal, however, their signs are opposed. This condition is given by:

$$
\begin{aligned}
\mathbf{H a}_{\mathbf{i}} & =\left[\begin{array}{c}
\mathbf{0}_{Q \times 1} \\
-\mathbf{H}_{l}
\end{array}\right] \mathbf{x}_{\mathbf{i}} \\
\mathbf{a}_{\mathbf{i}} & =\left(\mathbf{H}^{H} \mathbf{H}\right)^{-1} \mathbf{H}^{H}\left[\begin{array}{c}
\mathbf{0}_{Q \times 1} \\
-\mathbf{H}_{l}
\end{array}\right] \mathbf{x}_{\mathbf{i}}
\end{aligned}
$$

the ISI-free signal, in second line in (8), is obtained by taking the channel inverse of both sides.

2) The circularity-providing signal: The last $\mu$ time samples of $\mathbf{y}_{\mathbf{i}}$ in (7), which are added to the succeeding symbol, are equal to zero due to the self-ISI cancellation mechanism. This means that the AS-SEFDM, after traversing a multipath channel, is ISI-free. The received $i^{t h}$ AS-SEFDM symbol $\left(\mathbf{r}_{\mathbf{i}} \in \mathbb{C}^{Q \times 1}\right)$ is expressed by:

$$
\mathbf{r}_{\mathbf{i}}=\mathbf{H}_{u} \mathbf{x}_{\mathbf{i}}+\mathbf{z}_{\mathbf{i}}
$$

where $\mathbf{z}_{\mathbf{i}} \in \mathbb{C}^{Q \times 1}$ is the AWGN noise vector.

In conventional CP-SEFDM systems, the received signal is padded with zeros before being passed to the DFT process, as shown in step 7 in Fig. 1. This padded SEFDM signal lacks the circularity providing signal due to zero padding at the receiver before the DFT. Unlike CP-SEFDM, in ASSEFDM systems we estimate the circularity-providing signal and attach it to the received ISI-free SEFDM signal, as shown in Fig. 3. The estimation procedures are obtained using the following steps: (i) pad the received signal, 


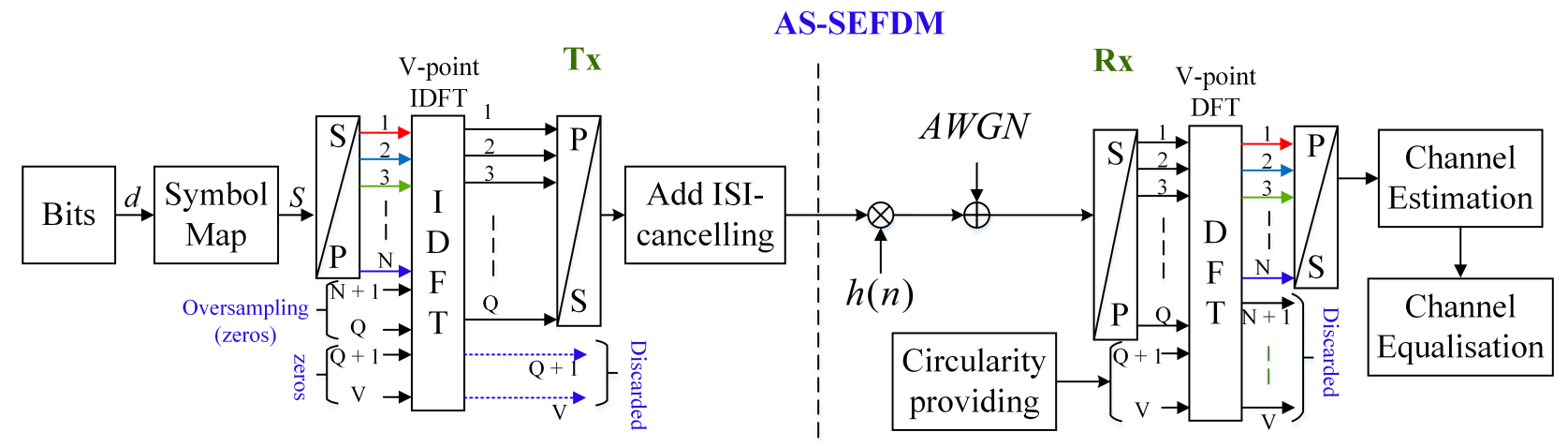

Fig. 3. A simplified block diagram of AS-SEFDM transceiver.

$\mathbf{r}_{\mathbf{i}} \in \mathbb{C}^{Q \times 1}$, to length $V$ time sample, $(i i)$ pass the padded SEFDM signal to $V$-point DFT $\left(\mathbf{F}^{H}\right)$, (iii) perform onetap equaliser at each subcarrier and decode the signal, and then $(i v)$ pass the output to $V$-point IDFT $(\mathbf{F})$ to obtain the signal in time-domain without the multipath channel effects. This can be written as:

$$
\tilde{\mathbf{r}}_{\mathbf{i}}=\mathbf{F} \boldsymbol{\lambda}^{-1} \mathbf{F}^{H}\left[\begin{array}{c}
\mathbf{r}_{\mathbf{i}} \\
\mathbf{0}_{(V-Q) \times 1}
\end{array}\right]
$$

where $\tilde{\mathbf{r}}_{\mathbf{i}} \in \mathbb{C}^{V \times 1}$ is the resulting signal vector in the timedomain. Out of the $V$ samples of $\tilde{\mathbf{r}}_{\mathbf{i}}$, only the first $Q$ samples, given as a vector $\tilde{\mathbf{x}}_{\mathbf{i}} \in \mathbb{C}^{Q \times 1}$, are passed to next step. The $\tilde{\mathbf{x}}_{\mathbf{i}}$ vector is convolved with the CIR, $\mathbf{h}$, and given by:

$$
\tilde{\mathbf{y}}_{\mathbf{i}}=\mathbf{h} * \tilde{\mathbf{x}}_{\mathbf{i}}=\mathbf{H} \tilde{\mathbf{x}}_{\mathbf{i}}=\left[\begin{array}{c}
\mathbf{H}_{u} \\
\mathbf{H}_{l}
\end{array}\right] \tilde{\mathbf{x}}_{\mathbf{i}}=\underbrace{\left[\begin{array}{c}
\mathbf{H}_{u} \\
\mathbf{0}_{\mu \times 1}
\end{array}\right]}_{1} \tilde{\mathbf{x}}_{\mathbf{i}}+\underbrace{\left[\begin{array}{c}
\mathbf{0}_{Q \times 1} \\
\mathbf{H}_{l}
\end{array}\right] \tilde{\mathbf{x}}_{\mathbf{i}}}_{2}
$$

Then, the last $(V-Q)$ samples of the resulting signal, part 2 in (11), are taken and attached to the received signal, given in (9), in the time-domain. Thus, (9) can be written as:

$$
\hat{\mathbf{r}}_{\mathbf{i}}=\left[\begin{array}{c}
\mathbf{H}_{u} \\
\mathbf{0}_{\mu \times 1}
\end{array}\right] \mathbf{x}_{\mathbf{i}}+\left[\begin{array}{c}
\mathbf{0}_{Q \times 1} \\
\mathbf{H}_{l}
\end{array}\right] \tilde{\mathbf{x}}_{\mathbf{i}}+\tilde{\mathbf{z}}_{\mathbf{i}}
$$

where $\tilde{\mathbf{z}}_{\mathbf{i}} \in \mathbb{C}^{V \times 1}$ is the AWGN noise vector. The resulting signal, $\hat{\mathbf{r}}_{\mathbf{i}} \in \mathbb{C}^{V \times 1}$ depicted in step 7 in Fig. 2, is passed to the DFT process as shown in Fig. 3.

\section{System Parameters And Performance EVALUATION}

In this section, we evaluate the performance of the proposed AS-SEFDM scheme versus the conventional CPSEFDM and CP-OFDM schemes. In Section III-A, we describe the system specifications and parameters; in Section III-B we evaluate the three schemes using error rate performance; after that we discuss the energy saving in Section III-C; then the transmission rate enhancement and the frequency bandwidth improvement are evaluated in Section III-D; and finally in Section III-E the peak-to-average power ratio (PAPR) performance is evaluated.
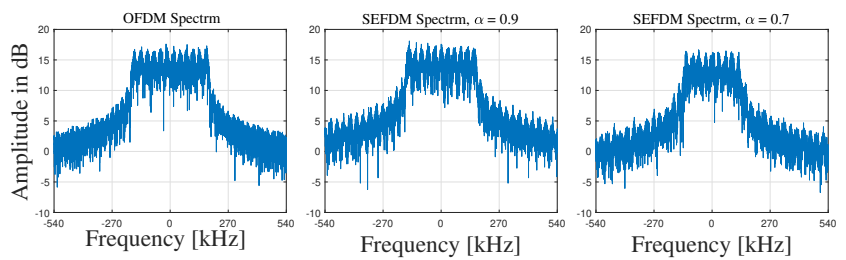

Fig. 4. The example spectrums of the transmitted OFDM signal and SEFDM signals with $\alpha=0.9$ and $\alpha=0.7$ respectively.

\section{A. Simulation Setting}

The system performance evaluation is carried out using computer simulations and it is based on the narrowbandinternet-of-things (NB-IoT) in the context of the 5G-NR systems. The system parameters are: the number of the active subcarriers is $N=12$ and the width of each subcarrier in the frequency-domain is equal to $30 \mathrm{KHz}$. The number of input bins to the IDFT/DFT processes is $Q=64$. The CP length is $\mu=16$ time samples. The message signal is modulated with quadrature phase shift keying (QPSK) while the pilot signals, which are used in channel estimation process, is modulated using binary phase shift keying (BPSK). The bandiwdth compression factors ranging between $\alpha=0.9 \sim 0.5$. The example sepctrums of OFDM signal and SEFDM signals based on the above system parameters are show in Fig. 4.

The error rate comparisons for AS-SEFDM, CP-SEFDM and $\mathrm{CP}-\mathrm{OFDM}$ are computed using the 5G-NR tapped delayed line (TDL) channel model of type (D). In this work, TDL-D channel model is used with 300 ns delay spread, $70 \mathrm{~Hz}$ maximum Doppler shift and $7 \mathrm{~dB} \mathrm{~K}$-factor.

\section{B. Bit Error Rate}

In this section, the BER performance for AS-SEFDM and CP-SEFDM will be evaluated and compared against our bench-mark, the conventional CP-OFDM. In this work, the equalised data is detected using sphere decoder. In order to examine the proposed AS-SEFDM scheme carefully we collect the BER for the following scenarios: $(i)$ using perfect channel estimation and $(i i)$ using realistic channel estimation. The first scenario of using perfect channel estimation provides the AS-SEFDM system capabilities of generating accurate ISI-cancelling signal and circularity-providing signal without taking into consideration the impairments 

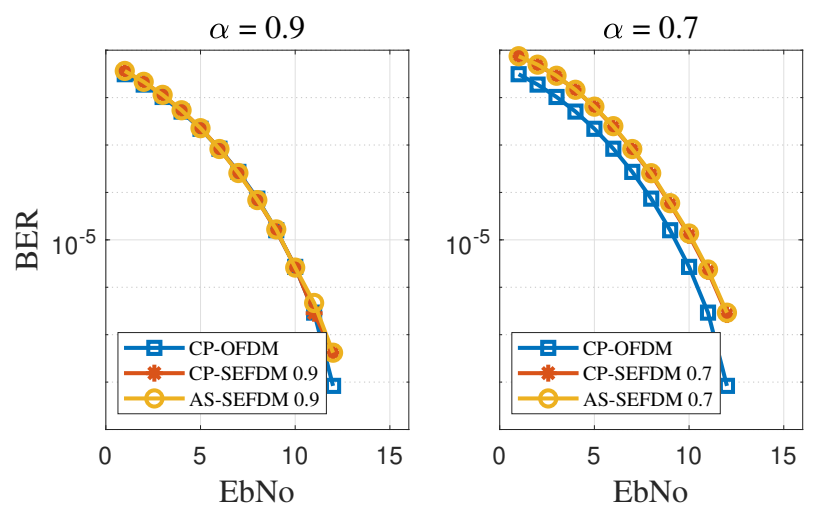

Fig. 5. BER results using perfect TDL-D channel information.

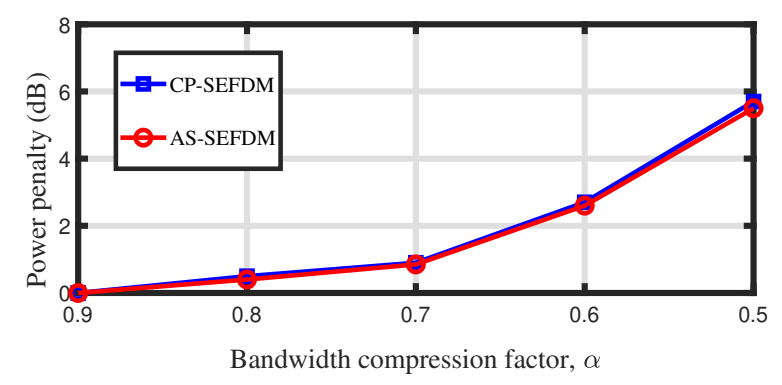

Fig. 6. Power Penalty of CP-SEFDM and AS-SEFDM.

coming from the channel estimation. On the other hand, the second scenario of using the realistic channel estimation aims to exam the performance of AS-SEFDM when noise is involved in the generating process of ISI-cancelling signal and circularity providing signal.

1) BER using perfect channel estimation: The perfect channel estimation's BER performance of AS-SEFDM, CPSEFDM and CP-OFDM is shown in Fig. 5. The BER performance of AS-SEFDM and CP-SEFDM with compression factor $\alpha=0.9$ and $\alpha=0.7$ show that AS-SEFDM scheme successfully eliminates the usage of $\mathrm{CP}$ while provides ISI-cancelling and circularity functions without introduce performance degradation compare with CP-SEFDM. From the same figure, we can report that SEFDM systems, in general, can offers up to $30 \%$ bandwidth saving at the cost of $1 \mathrm{~dB}$ in $E_{b} / N_{o}$.

Moreover, Fig. 6 shows the power penalty, which is defined as the power difference, measured in $(\mathrm{dB})$, between SEFDM and OFDM systems to have BER $=10^{-5}$. The figure also illustrate the performance of AS-SEFDM and CP-SEFDM for different values of bandwidth compression factor, $\alpha$. It is clear that using lower values of the $\alpha$ factor leads to degrades the error rate performance. This is expected, where the lower $\alpha$ factor the higher ICI among the subcarriers.

2) BER using realistic channel estimation: Fig. 7 shows the error rate performance collected using the realistic channel estimation. In general, the BER performance is worse than that of using perfect channel estimation. This is due to the impairments introduced by the inaccurate channel
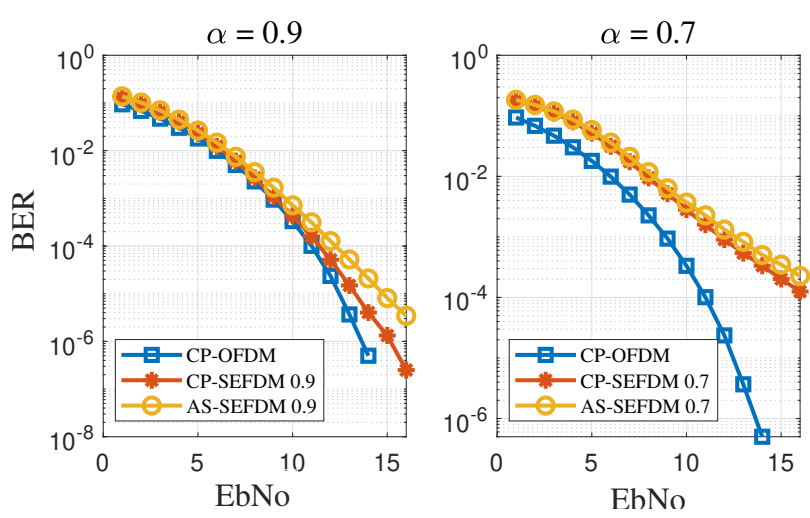

Fig. 7. BER results using TDL-D channel estimation.

estimation process. Besides that, the SEFDM schemes experience lower BER performance, i.e. higher BER degradation, this phenomenon becomes clearer at the higher $E_{b} / N_{o}$. The reason for such degradation in AS-SEFDM, comes from the inaccurate calculation of the AS signals, i.e. the ISIcancelling and the circularity-providing signals. This can be better understood by referring back to equations (8) and (10) in the previous section, where the two equations depends totally on the channel estimation for the signals generation.

Nevertheless, although the BER performance of the ASSEFDM is degraded in the realistic channel estimation situation, its BER performance is close to that of the CPSEFDM with the same respected $\alpha$ factors. This infer the successful operation of the newly proposed AS-SEFDM.

\section{Energy Saving and Latency Reduction}

As can be seen from Figures 5 and 7, the AS-SEFDM almost achieves same BER performance as the CP-SEFDM when perfect channel knowledge is known in transmitter and receiver, and presents slightly BER performance drop when the channel estimation errors are taken into account. This is a very economic cost for gaining the benefits of removing the CP parts between the SEFDM symbols. Using the simulation setting in this paper, the elimination of the 16 samples CP saves $23.8 \%$ of the power transmission and reduces the same proportion of latency. Together with the improved spectral efficiency brought by the spectral compression in SEFDM systems. Thus, the AS aided CP-free SEFDM system shows promising merits for the 5G-NR or beyond systems.

\section{Spectral Efficiency Enhancement}

The use of CP part in the OFDM and SEFDM systems degrades the spectral efficiency of the systems due to the transmission of redundant information in the $\mathrm{CP}$ part. Unlike CP-OFDM and CP-SEFDM, AS-SEFDM has the ability to overcome this spectral efficiency drawback with eliminating $\mathrm{CP}$, yet maintaining similar BER performance to that of SEFDM with CP. Table I indicates the frequency bandwidth required for signal transmission and the time needed for each symbol transmission for the three tested systems. It is clear that the two SEFDM schemes outperform the OFDM scheme, in terms of bandwidth utilisation, by the $\alpha$ factor. In 
TABLE I

SPECTRAL EFFICIENCY COMPARISONS

\begin{tabular}{|c|c|c|c|}
\hline Parameters & AS-SEFDM & CP-SEFDM & CP-OFDM \\
\hline \hline $\begin{array}{c}\text { Frequency } \\
\text { Bandwidth }\end{array}$ & $\alpha \times N \times \Delta f$ & $\alpha \times N \times \Delta f$ & $N \times \Delta f$ \\
\hline $\begin{array}{c}\text { Transmission } \\
\text { Time }\end{array}$ & $T$ & $T+T_{c p}$ & $T+T_{c p}$ \\
\hline $\begin{array}{c}\text { Spectral } \\
\text { Efficiency }\end{array}$ & $\frac{K / T}{\alpha \times N \times \Delta f}$ & $\frac{K /\left(T+T_{c p}\right)}{\alpha \times N \times \Delta f}$ & $\frac{K /\left(T+T_{c p}\right)}{N \times \Delta f}$ \\
\hline
\end{tabular}
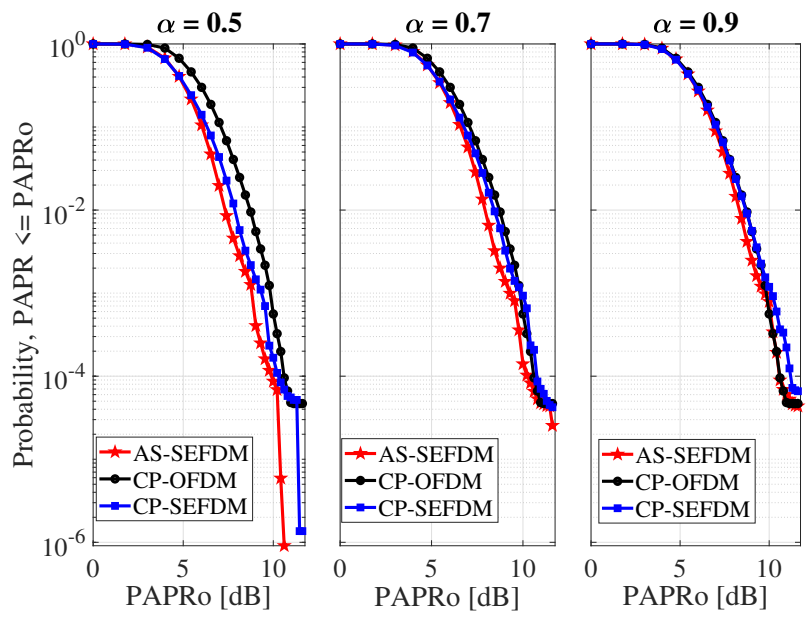

Fig. 8. PAPR of AS-SEFDM, CP-SEFDM and CP-OFDM.

addition, AS-SEFDM outperforms the OFDM and SEFDM with $\mathrm{CP}$, in terms of the transmission rate enhancement, by a factor of $\frac{T+T_{c p}}{T}$, where $T$ is the symbol duration and $T_{c p}$ is the time period of the CP part. Finally, the total spectral efficiency is given in the third row of the same table. $K$ indicates the number of bits transmitted per symbol.

\section{E. Peak-to-Average Power Ratio}

Fig. 8 shows the PAPR performance of the three tested systems. The evaluation is presented using complementary cumulative distribution function (CCDF), which is the probability of having PAPR values, at the transmitter side, exceeding a certain value of PAPR, denoted by PAPRo. From the figure, we can observe that lower $\alpha$ factor, in SEFDM, leads to a lower PAPR and this is due to the introduction of ICI among the subcarriers. In addition, AS-SEFDM shows further enhancement in the PAPR performance due to the ISI-cancelling signal, which is added at the transmitter, acts as a vector-perturbation and hence reduces the PAPR.

\section{CONCLUSION}

SEFDM systems compresses the frequency spacing between the subcarriers and hence offers bandwidth enhancement compared to the OFDM systems. However, CPSEFDM relies on the $\mathrm{CP}$ to mitigate the ISI effects at the receiver and therefore counterparts the spectral compression benefit. Furthermore, previous studies indicate that the use of CP with SEFDM breaks the circularity of the channel matrix and hence degrades the SEFDM system performance. The AS-SEFDM in this work eliminates the ISI by using transmitting AS signal and providing circularity on receiver. The simulation results show that the AS-SEFDM provide promising potential to save energy, reduce latency, improve spectral efficiency in future mobile communications. Following this work, experimental verification of AS-SEFMD and AS design for other high spectral efficiency multiple carrier schemes such as generalised frequency division multiplexing (GFDM), faster-than-Nyquist (FTN) and Truncated OFDM (TOFDM) will be exploited.

\section{ACKNOWLEDGEMENT}

Bo Tan would like to thank the sponsorship of the Profi 2 (No. 326654) funded by Academy of Finland.

\section{REFERENCES}

[1] A. Goldsmith, Wireless communications. Cambridge university press, 2005.

[2] G. T. . version 15.2.0 Release 15, "5G; Procedures for the 5G System," June 2018.

[3] G. T. . version 8.12.0 Release 8, "Evolved universal terrestrial radio access (E-UTRA) and evolved universal terrestrial radio access network (EUTRAN); overall description; stage 2 (release 8)," April 2010.

[4] E. Perahia and R. Stacey, Next generation wireless LANs: $802.11 n$ and $802.11 \mathrm{ac}$. Cambridge university press, 2013.

[5] X. Wang, P. Ho, and Y. Wu, "Robust channel estimation and isi cancellation for ofdm systems with suppressed features," IEEE Journal on Selected Areas in Communications, vol. 23, no. 5, 2005.

[6] J. M. Hamamreh, Z. E. Ankarali, and H. Arslan, "CP-less OFDM with alignment signals for enhancing spectral efficiency, reducing latency, and improving PHY security of 5G services," IEEE Access, vol. 6, pp. 63649-63 663, 2018.

[7] I. Kanaras, A. Chorti, M. R. D. Rodrigues, and I. Darwazeh, "Spectrally Efficient FDM Signals: Bandwidth Gain at the Expense of Receiver Complexity," in Proc. IEEE Int. Conf. Commun., June 2009.

[8] B. Yu et al., "Channel equalisation and data detection for SEFDM over frequency selective fading channels," IET Communications, vol. 12, no. $18,2018$.

[9] Z. Li et al., "Beyond $100 \mathrm{~Gb} / \mathrm{s}$ SEFDM Signal IM/DD Transmission Utilizing TDE With 20\% Bandwidth Compression," IEEE Communications Letters, vol. 23, no. 11, pp. 2017-2021, 2019.

[10] C. Jayawardena and K. Nikitopoulos, "G-multisphere: Generalizing massively parallel detection for non-orthogonal signal transmissions," IEEE Transactions on Communications, pp. 1-12, 2019.

[11] M. Ganji et al., "A Block-Based Non-Orthogonal Multicarrier Scheme," in 2019 IEEE Global Communications Conference (GLOBECOM). IEEE, 2019, pp. 1-6.

[12] S. Stainton et al., "Neural Network Equalisation and Symbol Detection for $802.11 \mathrm{p} \mathrm{V} 2 \mathrm{~V}$ Communication at $5.9 \mathrm{GHz}$," in IEEE Vehicular Technology Conference, vol. 91. IEEE, 2020.

[13] W. Ozan et al., "Zero padding or cyclic prefix: Evaluation for nonorthogonal signals," IEEE Communications Letters, 2020.

[14] S. Isam and I. Darwazeh, "Robust channel estimation for Spectrally Efficient FDM system," in Proc. 19th Int. Conf. Telecommun. (ICT), April 2012, pp. 1-6.

[15] W. Ozan et al., "Experimental SEFDM Pipelined Iterative Detection Architecture with Improved Throughput," in 2018 IEEE 87th Vehicular Technol. Conf. (VTC Spring), June 2018, pp. 1-5.

[16] S. Gorbunov and A. Rashich, "BER performance of SEFDM signals in LTE fading channels," in 2018 41st International Conference on Telecommunications and Signal Processing (TSP). IEEE, 2018.

[17] H. Ghannam and I. Darwazeh, "Robust Channel Estimation Methods for Spectrally Efficient FDM Systems," in Proc. IEEE 87th Veh. Technol. Conf. (VTC Spring), June 2018, pp. 1-6.

[18] T. Levanen et al., "Transparent Tx and Rx waveform processing for $5 \mathrm{G}$ new radio mobile communications," IEEE Wireless Communications, vol. 26, no. 1, pp. 128-136, Feb. 2019.

[19] S. Isam and I. Darwazeh, "Characterizing the intercarrier interference of non-orthogonal spectrally efficient FDM system," in 2012 8th International Symposium on Communication Systems, Networks \& Digital Signal Processing (CSNDSP). IEEE, 2012, pp. 1-5. 